\title{
A case of septic pulmonary embolism caused by urinary tract infection
}

Yohei Okada, Shigeyosi Kamata, Satoru Kawakami, Takumi Yamada

Department of Urology, Saitama Medical Center and Department of Urology, Soka City Hospital

A 56-year-old woman with diabetes mellitus (DM) presented at our department with intermittent low-grade fever, right-side back pain and general fatigue, having taken antibiotics (cefotiam) for the preceding two weeks as prescribed at another healthcare facility. Despite the absence of any cough or sputum production, chest X-ray revealed multiple nodular lesions in the peripheral lung fields (Figure-1). These nodules were not observed at the time of initial treatment at the referring hospital. Chest CT indicated multiple nodules with necrotic centers in the peripheral bilateral lung fields (Figure-2). Abdominal CT showed a swollen right kidney and diffuse abscesses (Figure-3). A diagnosis of septic pulmonary embolism accompanied by pyelonephrosis was made. Accordingly, an alternative antibiotic (cefotaxime) was

Figure 1 - A chest roentgenogram showed poorly marginated nodules in the bilateral peripheral lung fields.

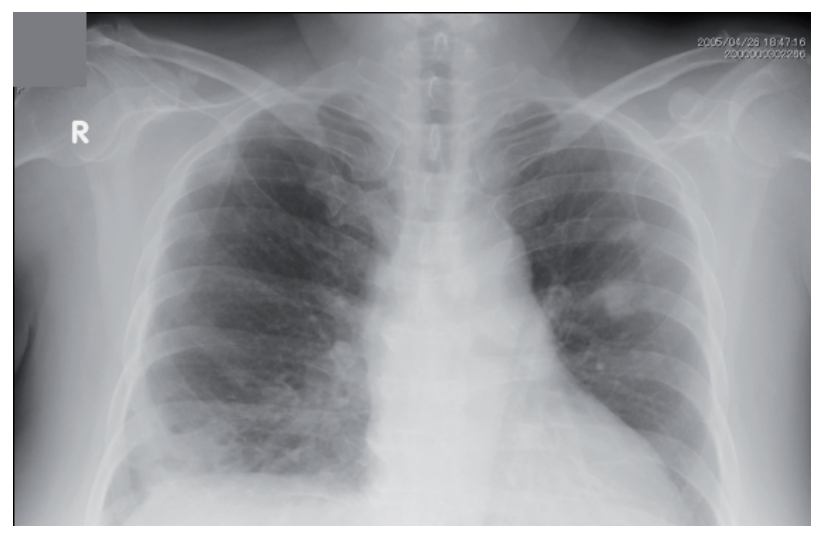

administered against sepsis. The multiple nodules in the lung and the diffuse renal abscesses simultaneously disappeared within two months after this course of highly potent anti-bacterial therapy. DM control was also accomplished by insulin therapy.

Septic pulmonary embolism has been defined as a lung embolism caused by a blood clot infected with any of several bacterial or fungal species. Typically, patients present with high-grade fever, productive cough, general malaise and hemoptysis. The presence of DM has an unfavorable impact on disease progression and prognosis. The most common radiographic findings are bilateral peripheral nodules with feeding vessels and cavity formations less than $3 \mathrm{~cm}$ in diameter (1). Septic pulmonary emboli are classified according to their source, i.e.

Figure 2 - Chest computed tomography (CT) showed multiple peripheral nodules with cavity formation in the bilateral lung fields.

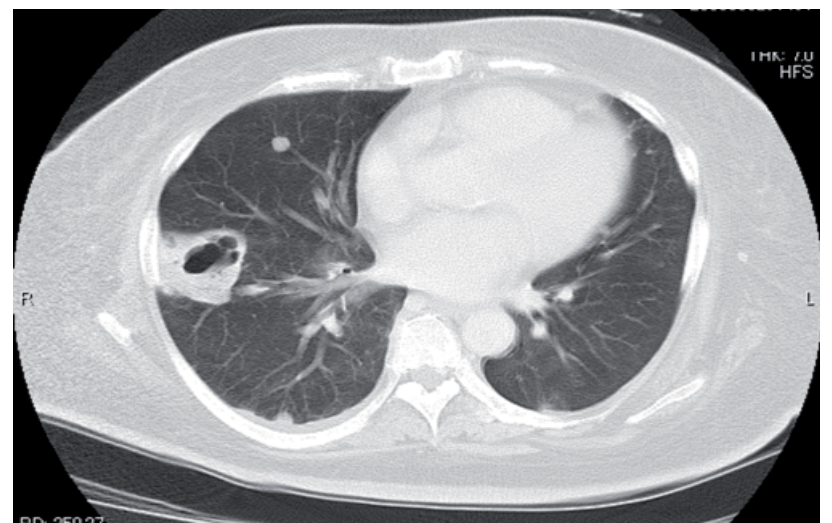


Figure 3 - Abdominal CT showed a swollen right kidney and multiple abscesses.

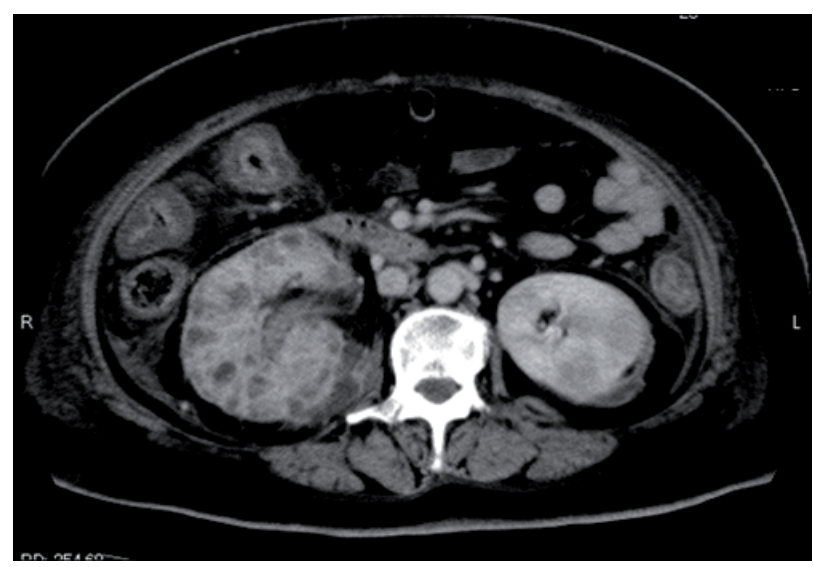

endocarditis, peripheral endogenous origin, or exogenous origin. Endogenous origins include peripheral abscesses without cardiac lesions (2). While septic pulmonary embolism occurring secondary to UTI appears to be rare $(3,4)$, it should be considered a possibility in DM patients with abnormal multiple nodules revealed in radiographic examinations. Although the mechanism underlying this disease is still unknown, it is well known that renal cell carcinoma can form tumor thrombi and metastasize to the lungs via the venous vessels rather than through lymphatic spread. Thus, the septic pulmonary embolism in our case was thought to have developed from an infected thrombus originating in a renal abscess derived from the patient's UTI.

\section{REFERENCES}

1. Iwasaki Y, Nagata K, Nakanishi M, Natuhara A, Harada H, Kubota $Y$, et al.: Spiral CT findings in septic pulmonary emboli. Eur J Radiol. 2001; 37: 190-4.

2. MacMillan JC, Milstein SH, Samson PC: Clinical spectrum of septic pulmonary embolism and infarction. J Thorac Cardiovasc Surg. 1978; 75: 670-9.

3. Kiwamoto T, Endo T, Sekizawa K: A case of septic pulmonary embolism induced by urinary tract infection. Nihon Kokyuki Gakkai Zasshi. 2004; 42: 89-93.

4. Takahashi S, Uehara T, Shima M, Takasugi S, Hashimoto $\mathrm{K}$, Itoh N: Septic pulmonary embolism caused by Candida albicans after treatment for urinary multidrug-resistant Pseudomonas aeruginosa. J Infect Chemother. 2008; 14: 436-8.

Correspondence address: Dr. Y. Okada Department of Urology, Saitama Medical Center 1981 Kamoda, Kawagoe, Saitama, Japan Fax: + 8149 228-3673 E-mail: okada@saitama-med.ac.jp 